Zhaoyang Duan, Terrance Wilms, Peter Neubauer, Costas Kravaris, Mariano Nicolas Cruz Bournazou

\title{
Model reduction of aerobic bioprocess models for efficient simulation
}

Journal article | Accepted manuscript (Postprint)

This version is available at https://doi.org/10.14279/depositonce-9723

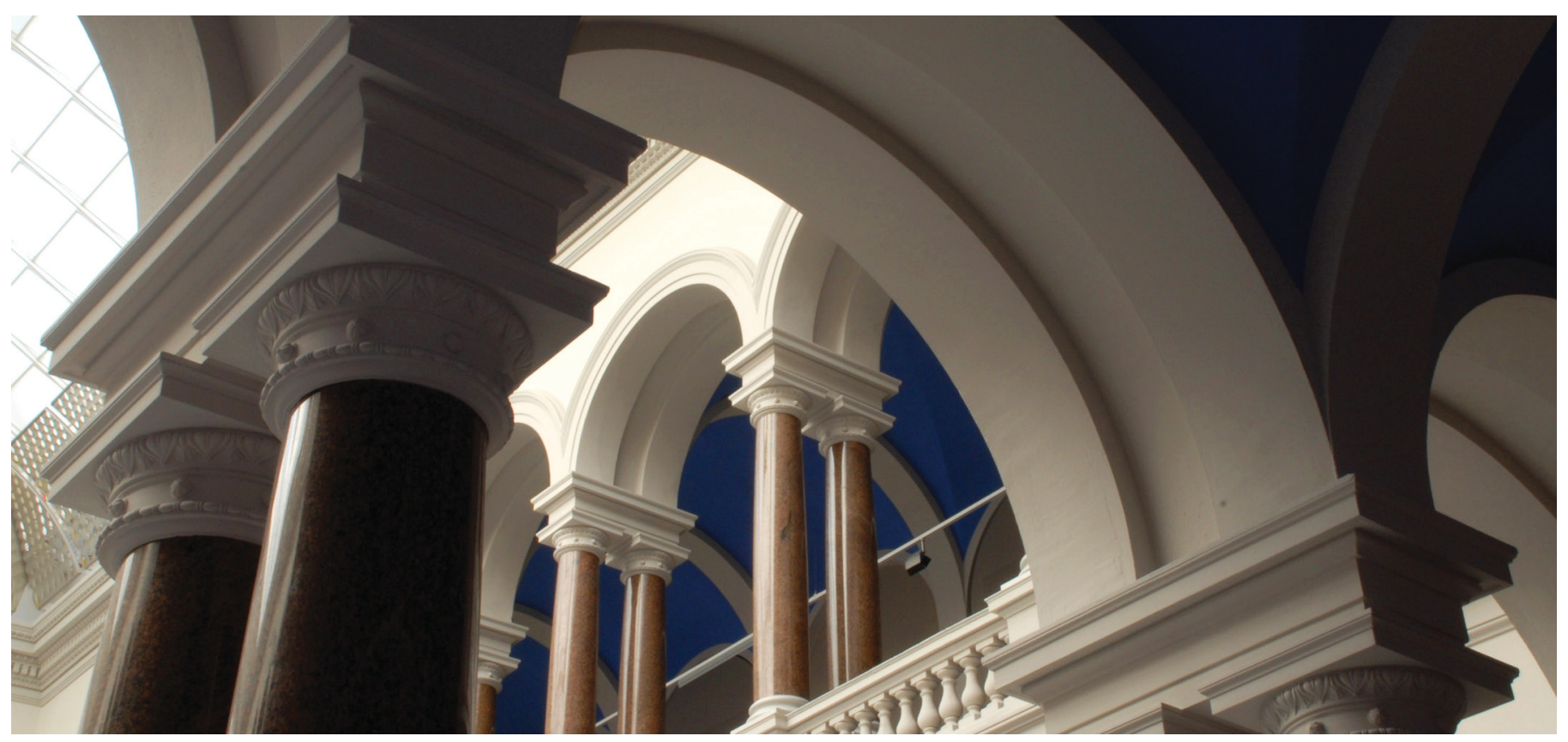

Duan, Z., Wilms, T., Neubauer, P., Kravaris, C., \& Cruz Bournazou, M. N. (2020). Model reduction of aerobic bioprocess models for efficient simulation. Chemical Engineering Science, 217, 115512. https://doi.org/10.1016/j.ces.2020.115512 


\title{
This accepted manuscript version is made available under the CC-BY-NC-ND 4.0 license http://creativecommons.org/licenses/by-nc-nd/4.0/
}

\section{Model reduction of aerobic bioprocess models for efficient simulation}

\author{
Zhaoyang Duan ${ }^{\mathrm{a}}$, Terrance Wilms ${ }^{\mathrm{b}}$, Peter Neubauer ${ }^{\mathrm{c}}$, Costas Kravaris ${ }^{\mathrm{a}, *}$, \\ Mariano Nicolas Cruz Bournazou d,e \\ ${ }^{a}$ Artie McFerrin Department of Chemical Engineering, Texas A $6 M$ University, College \\ Station, TX 77843, USA \\ ${ }^{b}$ Chair of Measurement and Control, Technical University Berlin, Seer. ER 2-1, \\ Hardenbergstrae 36a, 10623 Berlin, Germany \\ ${ }^{c}$ Department of Bioprocess Engineering, Institute of Biotechnology, Technische Universität \\ Berlin, D-13355 Berlin, Germany \\ ${ }^{d}$ DataHow AG, Zurich Switzerland \\ ${ }^{e}$ Institute of Chemical and Bioengineering, Department of Chemistry and Applied \\ Biosciences, ETH Zrich, Switzerland
}

\begin{abstract}
Owing to the increasing demand for large scale and high efficiency in manufacturing processes, computer aided tools for process operation and control are rapidly gaining popularity. An important state variable in aerobic processes is the dissolved oxygen, which can be easily measured online and is an important indicator of the metabolic activity. However, due to the fast kinetics of the oxygen transfer, dynamical models describing aerobic bioprocesses tend to be highly stiff. This can lead to significant numerical problems hampering its use for fixed step discretization methods and computationally costly applications such as computer fluid dynamics. In this work we use the slow-motion invariant manifold and the quasi steady state assumption methods to eliminate the differential equation describing the dissolved oxygen (the fast mode). By doing this, the tractability of the model is significantly increased with a neglectable loss in description power. The reduced model is also useful for simplifying the observer design problems, which is demonstrated by a state and parameter estimation
\end{abstract}

\footnotetext{
* Corresponding author

Email addresses: zy.duan@tamu.edu (Zhaoyang Duan), terrance.wilms@tu-berlin.de (Terrance Wilms), peter.neubauer@tu-berlin.de (Peter Neubauer), kravaris@tamu.edu (Costas Kravaris), nicolas.cruz@tu-berlin.de (Mariano Nicolas Cruz Bournazou)
} 
example at the end of the work.

Keywords: Model Reduction, Dissolved Oxygen Tension, Aerobic, Nonlinear Dynamics, Observer

\section{Introduction}

A rapid shift in industry towards more sustainable manufacturing processes is desperately needed to meet the current environmental challenges. Biotechnology offers attractive alternatives to traditional chemical engineering pro-

5 cesses for the production of pharmaceuticals (e.g. antibodies and insulin [1, 2] ), biodegradable materials, and renewable fuels [3].

Nevertheless, bioprocesses typically lie behind chemical engineering solutions in terms of costs, developmental times, process efficiency, and reliability. For this reason, it is essential to tailor and apply existing process systems engineering (PSE) tools for process design, optimization and control [4, 5, 6, 7, 8, 9, But for this, an accurate and tractable mathematical description of the bioprocess is required. Once available, mathematical models can be used from regulatory agencies support as in Quality by Design (QbD) [10], over model-based process monitoring (e.g. soft sensors, nonlinear observers) and control [11, up to plant 15 optimization [12, 13, and to plant-wide optimization [14]. And even though it is true that it is difficult to mathematically describe the dynamics of living organisms, macro-kinetic growth models offer a good trade-off between descriptive power and model tractability for bioprocesses [15, 16, 17].

The applicability of advanced model-based methods depends on the properties of the models used, such as accuracy, robustness, and identifiability. Stiffness, for example, increases the computational burden and instability of the numerical integration. Differential equation systems that have both very fast and very slow modes will require very small discretization steps to catch all changes in the system and need long time spans to allow slow modes to come to equilibrium. Stiffness is especially inconvenient for fixed step discretization methods (e.g. full discretization) and large systems of partial differential equations (e.g. 
computational fluid dynamics). In aerobic bioprocesses, the big difference in dynamics between physicochemical phenomena and biological reaction can be very challenging. The dissolved oxygen, for instance, reveals important process insights, but is prone to cause stiffness due to its fast kinetics.

There are a number of methods that can be applied to reduce the order of chemical and biochemical models 18 . Some of them are easy to implement and thereby widely used, such as the quasi-steady-state assumption (QSSA) method [19, 20, and singular perturbation method [21, 22. However, due to the fact that these methods render some non-vanishing errors between the reduced model and the original one, the performance of the reduced model cannot be guaranteed for those applications that are sensitive to such errors. An alternative method to overcome this is the slow-motion invariant manifold method which can guarantee the convergence of the reduced model [23]. The invariant manifold method

40 has been successfully applied to the fields of anaerobic digestion $24,25,26$ 27. and metabolic models [28, and is also widely used for the reduction of general chemical kinetics models 22, 30, 31. In this study, both the invariant manifold and the quasi-steady-state assumption methods are applied in search for a tractable but accurate description of aerobic bioprocesses. We demonstrate

45 that it is possible to increase the tractability of aerobic bioprocess models with a minimal loss in accuracy.

We will first study the model reduction problem for aerobic processes. In section 2 , we analyze the dynamic properties of a basic 3 -state aerobic system to demonstrate why model reduction is meaningful, and use the detailed $E$. coli model as a numerical example. In section 3, both continuous and fed-batch process models will be reduced with the slow-motion invariant manifold method, in comparison to the simpler quasi-steady-state approximation approach. After the model reduction, in section 4, we will work on an example to show how the reduced model could simplify the observer design problem to estimate the state 55 and parameter. 


\section{Aerobic Model and System Properties}

In aerobic processes, substrate is consumed by biomass in the presence of oxygen. Other organic and inorganic matters may also be involved in the process, such as acetate, enzyme, etc. For representative purposes, we will start with a basic aerobic model consisting of 4 states: biomass concentration $(X)$ in $[\mathrm{g} / \mathrm{L}]$, substrate concentration $(S)$ in $[\mathrm{g} / \mathrm{L}]$, dissolved oxygen tension $(D O T)$ in $[\%]$ and the volume of culture medium $(V)$ in $[\mathrm{L}]$ :

$$
\begin{aligned}
\frac{d S}{d t} & =-\frac{F}{V}\left(S-S_{\text {in }}\right)-q_{s} X \\
\frac{d X}{d t} & =-\frac{F}{V} X+\mu X \\
\frac{d D O T}{d t} & =K_{L} a\left(D O T^{*}-D O T\right)-H q_{o} X \\
\frac{d V}{d t} & =F-F_{\text {out }}
\end{aligned}
$$

$F$ and $F_{\text {out }}$ are the inlet and outlet flow rates. The substrate is fed to the system with a concentration of $S_{i n}$. In the DOT equation, $K_{L} a$ is the mass transfer coefficient for oxygen, $D O T^{*}$ is the percentage of dissolved oxygen saturation and $H$ is the Henry coefficient.

In real practice, the process is usually operated under substrate-limited conditions, where the oxygen is in excess. It is reasonable to assume that DOT is kept above the $20 \%$ level all the time and thereby the oxygen uptake rate $q_{o}$ is independent of DOT. Then the biomass growth rate $\mu$, the substrate and oxygen uptake rates $q_{s}$ and $q_{o}$ are functions of the substrate concentration only:

$$
\begin{aligned}
q_{s} & =q_{s \max } \frac{S}{S+K_{S}} \\
\mu & =Y_{x} q_{s} \\
q_{o} & =Y_{o / s} \cdot\left(q_{s}-\mu \cdot \frac{C_{X}}{C_{S}}\right)
\end{aligned}
$$

The definition for other coefficients are given in Table 1, as well as a set of representative parameter values to be used in the following sections, which is based on Enfors' book [16] and our experimental practices. As $C_{x}$ and $C_{s}$ in (2) can be treated as constant, $q_{o}$ is then proportional to $q_{s}$. Thus, we can define 
65 an overall DOT to substrate yield coefficient $Y_{o}=Y_{o / s}-Y_{o / s} Y_{x} C_{X} / C_{S}$ and rewrite as $q_{o}=Y_{o} q_{s}$.

Table 1: Parameter values

$\begin{array}{llll}S_{i n} & \text { Organic substrate concentration in feed } & 20 & \mathrm{~g} / \mathrm{L} \\ q_{s \max } & \text { Maximum aerobic substrate uptake rate } & 1.5 & \mathrm{~g} / \mathrm{gh} \\ K_{s} & \text { Half-saturation constant for organic substrates } & 0.05 & \mathrm{~g} / \mathrm{L} \\ D O T^{*} & \text { Percentage of dissolved oxygen saturation } & 100 & \% \\ K_{L} a & \text { Mass transfer coefficient for oxygen } & 800 & \mathrm{~h}^{-1} \\ Y_{x} & \text { Specific aerobic yield for substrate } & 0.56 & \mathrm{~g} / \mathrm{g} \\ Y_{o / s} & \text { Specific yield of DOT to substrate } & 1.217 & \% / \mathrm{g} \\ H & \text { Henry coefficient } & 14000 & \\ D & \text { Dilution rate } & 0.4 & \mathrm{~h}-1 \\ C_{s} & \text { Carbon content of substrate } & 0.487 & \mathrm{gC} / \mathrm{gX} \\ C_{x} & \text { Carbon content of biomass } & 0.391 & \mathrm{gC} / \mathrm{gX}\end{array}$

There could be three operating conditions depending on the inlet and outlet flows: batch, fed-batch and continuous operations. The batch reactor has neither inflow nor outflow: $F=F_{\text {out }}=0$. The fed-batch reactor has only inflow but no outflow: $F_{\text {out }}=0$. In continuous operation, the rate of the inlet flow equals to that of the outflow: $F=F_{\text {out }} \neq 0$. Therefore, the volume of culture remains constant in both batch and continuous reactors, and the state $V$ in model (1) can be dismissed for these operations. Particularly, the continuous reactor model can be written as:

$$
\begin{aligned}
\frac{d S}{d t} & =-D\left(S-S_{i n}\right)-q_{s} X \\
\frac{d X}{d t} & =-D X+\mu X \\
\frac{d D O T}{d t} & =K_{L} a\left(D O T^{*}-D O T\right)-H q_{o} X
\end{aligned}
$$

where $D=F / V$ represents the dilution rate and is defined as the ratio of inlet flow rate over the culture medium volume. 
To study the properties of aerobic systems, the continuous process would be a good starting point. For the model (3), there are two steady states: one is a trivial steady state, where the biomass is washed out; and the other non-trivial steady state can be calculated as:

$$
\begin{aligned}
S_{s} & =\frac{-D K_{s}}{-Y_{x} q_{s \max }+D} \\
X_{s} & =Y_{x}\left(S_{i n}-S_{s}\right) \\
D O T_{s} & =D O T^{*}-\frac{Y_{o / s} H D\left(S_{i n}-S_{s}\right)}{K_{L} a}
\end{aligned}
$$

By linearizing model (3) around this steady state, we get the following expression:

$$
\left[\begin{array}{c}
\dot{S} \\
\dot{X} \\
D \dot{O} T
\end{array}\right]=\left[\begin{array}{ccc}
-D-\left.X_{s} \frac{d q_{s}}{d S}\right|_{s . s .} & -\frac{D}{Y_{x}} & 0 \\
-\left.Y_{x} X_{s} \frac{d q_{s}}{d S}\right|_{s . s .} & 0 & 0 \\
-\left.H Y_{o} X_{s} \frac{d q_{s}}{d S}\right|_{s . s} & -\frac{Y_{o}}{Y_{x}} D H & -K_{L} a
\end{array}\right]\left[\begin{array}{c}
S-S_{s} \\
X-X_{s} \\
D O T-D O T_{s}
\end{array}\right]
$$

and the corresponding eigenvalues:

$$
\begin{aligned}
& \lambda_{1}=-D \\
& \lambda_{2}=-K_{L} a \\
& \lambda_{3}=-\frac{q_{s \max } K_{s} X_{s}}{\left(S_{s}+K_{s}\right)^{2}}=\frac{Y_{x} q_{s \max }-D}{Y_{x} q_{s \max } K_{s}}\left(S_{i n} Y_{x} q_{s \max }-S_{i n} D-D K_{s}\right)
\end{aligned}
$$

Apparently, all three eigenvalues are negative and this is a stable steady state. It can be seen that the eigenvalue $\lambda_{1}$ equals to the negative dilution rate. The value of $\lambda_{2}$ is the mass transfer coefficient for oxygen $-K_{L} a$, which is usually a very large number such that $K_{L} a>D$ and $\lambda_{3}$ is also small compared to $K_{L} a$. Since these eigenvalues are of different orders of magnitude, the process is comprised of fast and slow dynamics, with $\lambda_{2}=-K_{L} a$ to be the fast eigenvalue, ${ }_{75} \lambda_{1}=-D$ and $\lambda_{3}$ related to reaction dynamics to be the slow. This spectral gap is more explicit given the numbers of eigenvalues calculated with the parameters in Table 1; $\lambda_{1}=-0.4, \lambda_{2}=-800$ and $\lambda_{3}=-91.98$.

Note that the linearized matrix in (4) is a lower block triangular matrix, with $-K_{L} a$ being the right bottom block, which is the value of the fast eigenvalue 
$\lambda_{2}$. The left upper block governs the dynamics of the first two states $S$ and $X$ , with only slow eigenvalues, and therefore they are the slow states. And the state $D O T$ is the fast state that associates with the fast eigenvalue $\lambda_{2}=-K_{L} a$. Consequently, the entire process has serial structure, with the slow dynamics followed by the fast dynamics.

The physiology of $E$. coli, which has been described mathematically and applied in various studies $[8,32,33$, can be used as an example to demonstrate these dynamic properties in a specific application. An E. coli model can be written as:

$$
\begin{aligned}
\frac{d S}{d t} & =-\frac{F}{V}\left(S-S_{\text {in }}\right)-q_{s} X \\
\frac{d X}{d t} & =-\frac{F}{V} X+\mu X \\
\frac{d A}{d t} & =-\frac{F}{V} A+q_{A} X \\
\frac{d D O T}{d t} & =K_{L} a\left(D O T^{*}-D O T\right)-q_{o} X H \\
\frac{d V}{d t} & =F-F_{\text {out }}
\end{aligned}
$$

85 This dynamic model is comprised of five ODEs describing five state variables, namely biomass $X$, extracellular concentrations of substrate (glucose) $S$, acetate $A$, dissolved oxygen DOT, and culture volume $V$. They are modelled in a standard form, which can be easily transformed to a continuous, fed-batch or batch process model.

The auxiliary algebraic equations, coupled with the ODEs to form the kinetic model, are listed as follows; they describe the biomass formation, and the 
intracellular interactions relating substrate, oxygen and acetate consumption:

$$
\begin{aligned}
q_{s} & =\frac{q_{s m a x}}{1+\frac{A}{K_{i a}}} \cdot \frac{S}{S+K_{s}} \\
q_{s o f} & =\frac{P_{\text {Amax }} q_{s}}{q_{s}+K_{a p}} \\
q_{s o x} & =\left(q_{s}-q_{s o f}\right) \\
p_{A} & =q_{s o f} Y_{a s} \\
q_{s A} & =\frac{q_{\text {Amax }}}{1+\frac{q_{s}}{K_{i s}}} \frac{A}{A+K_{s a}} \\
q_{A} & =p_{A}-q_{s A} \\
q_{o} & =\left(q_{\text {sox }}-q_{m}\right) Y_{\text {os }}+q_{s A} * Y_{\text {oa }} \\
\mu & =\left(q_{\text {sox }}-q_{m}\right) Y_{\text {em }}+q_{\text {sof }} * Y_{\text {xsof }}+q_{s A} * Y_{x a}
\end{aligned}
$$

90 Note that under the assumption that $D O T>20 \%$, the kinetics functions are still independent of DOT. An elaborate description of this model and parameter values can be found in the publication of Anane et. al 8 .

For a continuous process with $D=0.15 \mathrm{~h}^{-1}, S_{i n}=20 \mathrm{~g} / \mathrm{L}$ and $K_{L} a=800$, the non-trivial steady state can be calculated numerically as:

$$
S_{s}=0.0273 \quad X_{s}=11.37 \quad A_{s}=0.0296 \quad D O T_{s}=38.67
$$

After linearization around the steady state, the state linear matrix is

$$
\left[\begin{array}{cccc}
-63.37 & -0.2634 & 2.36 & 0 \\
28.89 & 0 & 3.728 & 0 \\
13.02 & 0.0004 & -9.042 & 0 \\
-1100000 & -4315 & -22955 & -800
\end{array}\right]
$$

And the eigenvalues around the steady state are calculated as

$$
\lambda_{1}=-800 \quad \lambda_{2}=-63.81 \quad \lambda_{3}=-8.445 \quad \lambda_{4}=-0.1512 ;
$$

It is evident that the system (5), even with more detailed kinetics, is comprised of both fast $\left(\lambda_{1}\right)$ and slow $\left(\lambda_{2}, \lambda_{3}\right.$ and $\left.\lambda_{4}\right)$ eigenvalues. Similarly to the ${ }_{95}$ previous basic system (3), the linear state matrix is also block lower triangular. 
Therefore, it can be concluded that DOT is the fast state that corresponds to the fast eigenvalue $\left(-K_{L} a\right)$ in the $E$. coli model.

Based on this fact, it's meaningful to properly eliminate the very fast dynamics associated with the state DOT in dynamic model (3), so that the stiffness issue can be avoided when applying these model to biology and bioengineering applications.

For fed-batch operations, the system is different as there is no steady state and it is meaningless to study their eigenvalues. However, inspired by the idea from continuous processes, we will still apply similar approaches to probe the fed-batch models, and then show that the reduced model is also valid for the fed-batch case as long as $K_{L} a$ is large.

\section{Model Reduction}

It has been shown in section 2 that the continuous aerobic process is comprised of fast and slow modes. Therefore, the models are eligible for further simplification by assuming the fast dynamics to be instantaneous and approximate the fast state as a function of other slow states. One approach for implementation is to find the slow-motion invariant manifold of the system and use it to approximate the fast state. A detailed tutorial and explanation about this method can be found in some previous work $22,24,27$.

Another widely used, simple but less accurate approach is the QSSA method. It assumes that the fast state reaches its steady state instantaneously, and thus approximating the differential equation of that state to be zero. One can directly solve the algebraic equations by setting the differential equations of the fast states to be zero.

120

In the following part, we will apply both methods to reduce the order of continuous and fed-batch models, and compare the results. 


\subsection{Continuous processes}

Consider a dynamic system of the form:

$$
\begin{aligned}
\frac{d x_{s}}{d t} & =F_{s}\left(x_{s}\right) \\
\frac{d x_{f}}{d t} & =F_{f}\left(x_{s}, x_{f}\right)
\end{aligned}
$$

with $x_{s}$ and $x_{f}$ being the vectors of slow and fast states, where the slow dynamics $F_{s}$ are followed by the fast dynamics $F_{f}$. The fast states can then be approximated by the slow-motion invariant manifold $x_{f}=T\left(x_{s}\right)$ where $T\left(x_{s}\right)$ satisfies the following invariance equation 23

$$
\frac{\partial T\left(x_{s}\right)}{\partial x_{s}} F_{s}\left(x_{s}, T\left(x_{s}\right)\right)=F_{f}\left(x_{s}, T\left(x_{s}\right)\right)
$$

In the basic continuous aerobic digestion model (1), the slow-motion invariant manifold $T(S, X)$ can be used to approximate the fast state $D O T$, and it should satisfy

$$
\begin{aligned}
\frac{\partial T(S, X)}{\partial X}(-D X+\mu(S) X)+\frac{\partial T(S, X)}{\partial S}\left[D\left(S_{i n}-S\right)-q_{s}(S) X\right] \\
\quad=K_{L} a\left(D O T^{*}-T(S, X)\right)-H q_{o}(S) X
\end{aligned}
$$

This is a first order nonlinear partial differential equation, whose exact solution is difficult to find in closed form. But an asymptotic solution can be derived by using perturbation analysis. As $K_{L} a$ is a large number, it is evident that $\epsilon=1 / K_{L} a$ is a very small parameter that can be used for perturbation analysis. Dividing both sides of equation (7) by $K_{L} a$, and we have

$$
\begin{aligned}
\varepsilon \frac{\partial T(S, X)}{\partial X}(-D X+\mu(S) X)+\varepsilon \frac{\partial T(S, X)}{\partial S} & {\left[D\left(S_{\text {in }}-S\right)-q_{s}(S) X\right] } \\
& =D O T^{*}-T(S, X)-\varepsilon H q_{o}(S) X
\end{aligned}
$$

Or equivalently,

$$
\begin{gathered}
\varepsilon \frac{\partial T(S, X)}{\partial X}\left(-D X+Y_{x} q_{s \max } X \frac{S}{S+K_{S}}\right)+\varepsilon \frac{\partial T(S, X)}{\partial S}\left[D\left(S_{i n}-S\right)-q_{s \max } X \frac{S}{S+K_{S}}\right] \\
=D O T^{*}-T(S, X)-\varepsilon Y_{o} q_{s \max } H X \frac{S}{S+K_{S}}
\end{gathered}
$$


Expanding $T(S, X)$ in asymptotic series in terms of the small parameter $\epsilon$ :

$$
T(S, X)=P_{0}(S, X)+P_{1}(S, X) \cdot \varepsilon+P_{2}(S, X) \cdot \varepsilon^{2}+P_{3}(S, X) \cdot \varepsilon^{3} \ldots
$$

and substituting (9) into (8), and we get

$$
\begin{aligned}
& \left(\frac{\partial P_{0}}{\partial X} \varepsilon+\frac{\partial P_{1}}{\partial X} \varepsilon^{2}+\frac{\partial P_{2}}{\partial X} \varepsilon^{3}\right)\left[\left(-D X+Y_{x} q_{s \max } X \frac{S}{S+K_{S}}\right)\right] \\
& +\left(\frac{\partial P_{0}}{\partial S} \varepsilon+\frac{\partial P_{1}}{\partial S} \varepsilon^{2}+\frac{\partial P_{2}}{\partial S} \varepsilon^{3}\right)\left[D\left(S_{i n}-S\right)-q_{s \max } X \frac{S}{S+K_{S}}\right] \\
& =-\left(P_{0}+P_{1} \varepsilon+P_{2} \varepsilon^{2}+P_{3} \varepsilon^{3}\right)+\left(D O T^{*}-\varepsilon Y_{o} q_{s \max } H X \frac{S}{S+K_{S}}\right)
\end{aligned}
$$

We can solve for the unknown terms $P_{i}$ in 10 by matching the coefficients of the terms in $\epsilon$ from lower order to higher order. The result with up to the second order in $\epsilon$ is:

$$
\begin{aligned}
& P_{0}=D O T^{*} \\
& P_{1}=-Y_{o} H X q_{s}(S) \\
& P_{2}=q_{o} H(-D X+\mu(S) X)+Y_{o} X H \frac{d q_{s}}{d S}\left[-D\left(S-S_{i n}\right)+q_{s}(S) X\right]
\end{aligned}
$$

where

$$
\frac{d q_{s}}{d S}=q_{\text {smax }} \frac{K_{S}}{\left(S+K_{S}\right)^{2}}
$$

The slow motion invariant manifold for DOT can be expressed as

$$
\begin{aligned}
T(S, X) & =P_{0}(X, S)+P_{1}(X, S) \cdot \frac{1}{K_{L} a}+P_{2}(X, S) \cdot \frac{1}{K_{L} a^{2}}+O\left[\left(\frac{1}{K_{L} a}\right)^{3}\right] \\
& =D O T^{*}-\frac{X H Y_{o} q_{s \max }}{K_{L} a} \frac{S}{S+K_{s}} \\
& +\frac{1}{K_{L} a^{2}}\left\{q_{o} H(-D X+\mu(S) X)+Y_{o} X H \frac{d q_{s}}{d S}\left[-D\left(S-S_{i n}\right)+q_{s}(S) X\right]\right\} \\
& +O\left[\left(\frac{1}{K_{L} a}\right)^{3}\right]
\end{aligned}
$$

Therefore, the reduced model becomes

$$
\begin{aligned}
\frac{d S}{d t} & =-D\left(S-S_{i n}\right)-q_{s}(S) X \\
\frac{d X}{d t} & =-D X+\mu(S) X \\
D O T & =T(S, X)
\end{aligned}
$$


where

$$
\begin{aligned}
\mu(S) & =Y_{x / s} q_{s} \\
q_{s}(S) & =q_{s \max } \frac{S}{S+K_{S}}
\end{aligned}
$$

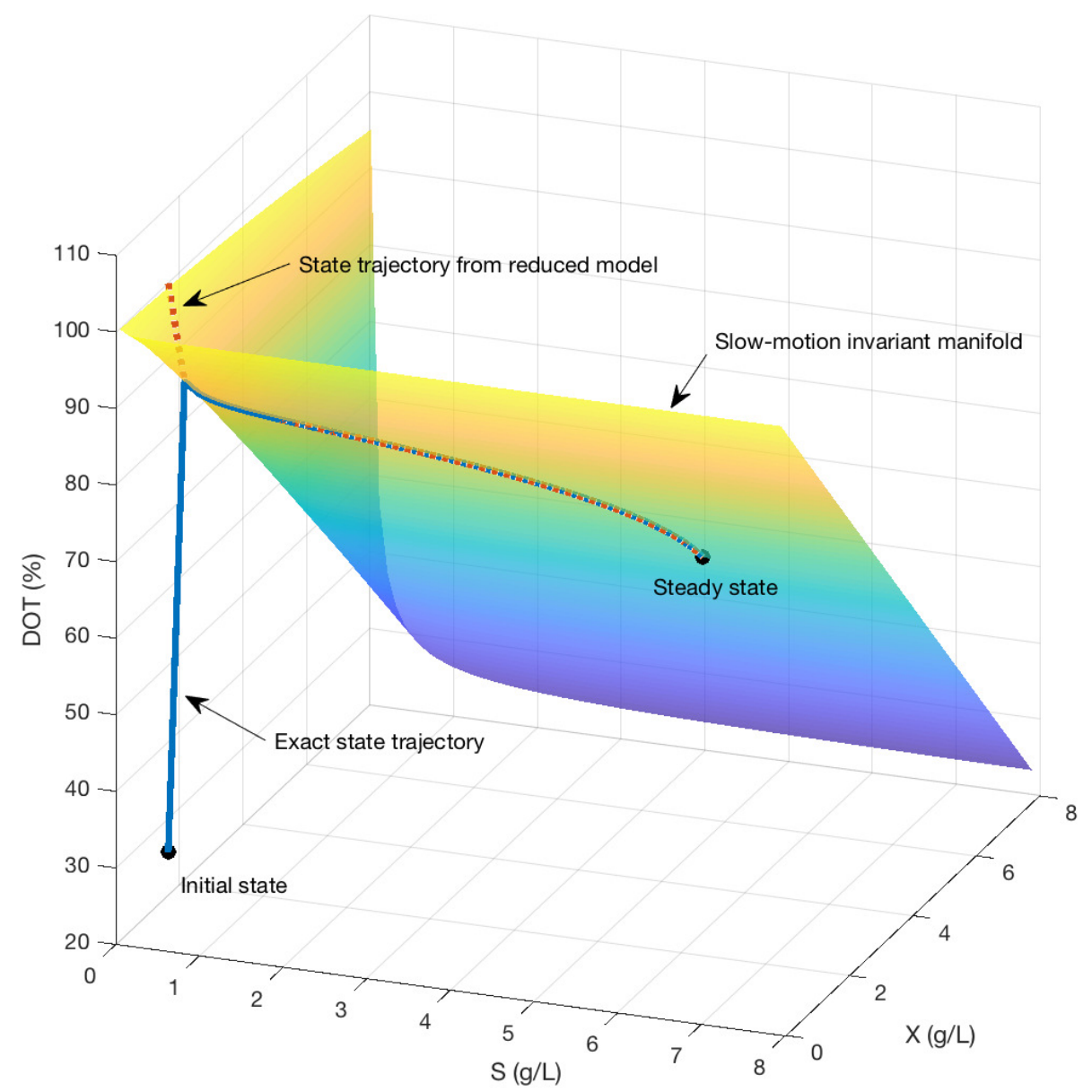

Figure 1: The evolution of real and reduced states on the invariant manifold

Figure 1 illustrates the approximation of the dynamics (3) through the reduced model 12 arising from the invariant manifold method. For an initial condition $S(0)=0.008 \mathrm{~g} / \mathrm{L}, X(0)=1.6 \mathrm{~g} / \mathrm{L}, \operatorname{DOT}(0)=25$, the blue line represents the dynamic response of the exact model (3), whereas the red dotted line of the reduced model 12 initialized at the same $S(0)$ and $X(0)$, but with 
$D O T(0)=T(S(0), X(0))$. The exact trajectory of the system involves an initial phase where the system approaches the invariant manifold very fast, and second phase where the system slowly moves along the invariant manifold and eventually reaches the steady state. The model reduction approximation involves projecting the initial state on the slow-motion invariant manifold, by essentially ignoring the very fast transient of approach to the invariant manifold. With the reduced system directly starting on the invariant manifold, all its trajectories will lie on it, as a consequence of the defining property of the invariant manifold 23]. So, the reduced model's state will involve an initial error that will rapidly decay at early stage and exponentially converge to zero.

Alternatively, one can use the QSSA method to find an approximation by assuming the state $D O T$ to be at steady state:

$$
\frac{d D O T}{d t}=K_{L} a\left(D O T^{*}-D O T\right)-q_{o}(S) X H \approx 0
$$

The result from quasi-steady-state approximation is

$$
D O T \approx D O T^{*}-\frac{X H Y_{o} q_{s \max }}{K_{L} a} \frac{S}{S+K_{s}}
$$

Comparing the results from slow motion invariant manifold method and the quasi-steady-state approximation, it can be seen that they are consistent with each other since the result from QSSA method matches the first two terms of the slow invariant manifold in 11 . As this result has an error of $O\left(1 / K_{L} a^{2}\right)$, when $K_{L} a$ is a very large number, this approximation is expected to show a good match to the real fast state. At the same time, it should also be noted that the higher order terms in the series solution for slow-motion invariant manifold could provide additional corrections to the leading terms, to render a more accurate approximation. One can decide how many terms to keep in the series solution, depending on the need for accuracy and calculation cost.

For the continuous operation of E. coli model (5), a similar model reduction approach can be performed. The asymptotic series expansion of the slow-motion 
invariant manifold up to the second order term can be calculated as:

$$
\begin{aligned}
T(S, X, A)= & D O T^{*}+\frac{1}{K_{L} a}\left(-X H q_{o}\right)+\frac{1}{K_{L} a^{2}}\left\{q_{o} H(-D X+\mu X)\right. \\
& \left.+X H \frac{\partial q_{o}}{\partial S}\left[D\left(S_{i n}-S\right)-q_{s} X\right]+X H \frac{\partial q_{o}}{\partial A}\left(-D A+q_{a} X\right)\right\} \\
& +O\left[\left(\frac{1}{K_{L} a}\right)^{3}\right]
\end{aligned}
$$

The QSSA result is again the same with the first two terms in series expansion for the slow-motion invariant manifold.

To verify the accuracy of the reduced model, we simulate the basic aerobic process in Figure 2 and the E. coli process in Figure 3 . At time 2 and 4 for the basic aerobic process, and at time 10 and 20 for the $E$. coli process, $K_{L} a$ is changed from 800 to 1000 and 900, as a result of some step change in oxygen flow rate and/or stirrer speed, to perturb the system. It can be observed from the graphs in both figures that the reduced models are in good agreement with the detailed ones most time except for the very fast changing period with peaking mismatch. The second graph, which depicts the error, clearly indicates these peaks, for whenever $K_{L} a$ value changes. The peaking issues at changing points are due to the elimination of the fast modes in reduced models, and thereby the reduced models are not capable of capturing the very fast dynamics when system undergoes a sudden change. In the third graph, which is a zoom-in view for the errors, the small size of error of orange lines indicates that even the result from QSSA method (corresponding to the first two terms in equation 11) is with satisfactory accuracy, but the presence of second order term (red lines) makes the reduced model even more precise. But whenever $K_{L} a$ value changes, there are noticeable peaking errors for both reduced models.

As a conclusion, for the typical aerobic systems, while the slow invariant manifold method guarantees the good accuracy and convergence of the reduced model, the result from QSSA method is also fairly accurate as long as $K_{L} a$ is very large. However, the result from QSSA method may not always be accurate enough for systems with moderately large $K_{L} a$. For example, with $K_{L} a=30$, $Y_{o}=0.107$ and $S_{i n}=10 \mathrm{~g} / \mathrm{L}$, running a new simulation leads to the results 

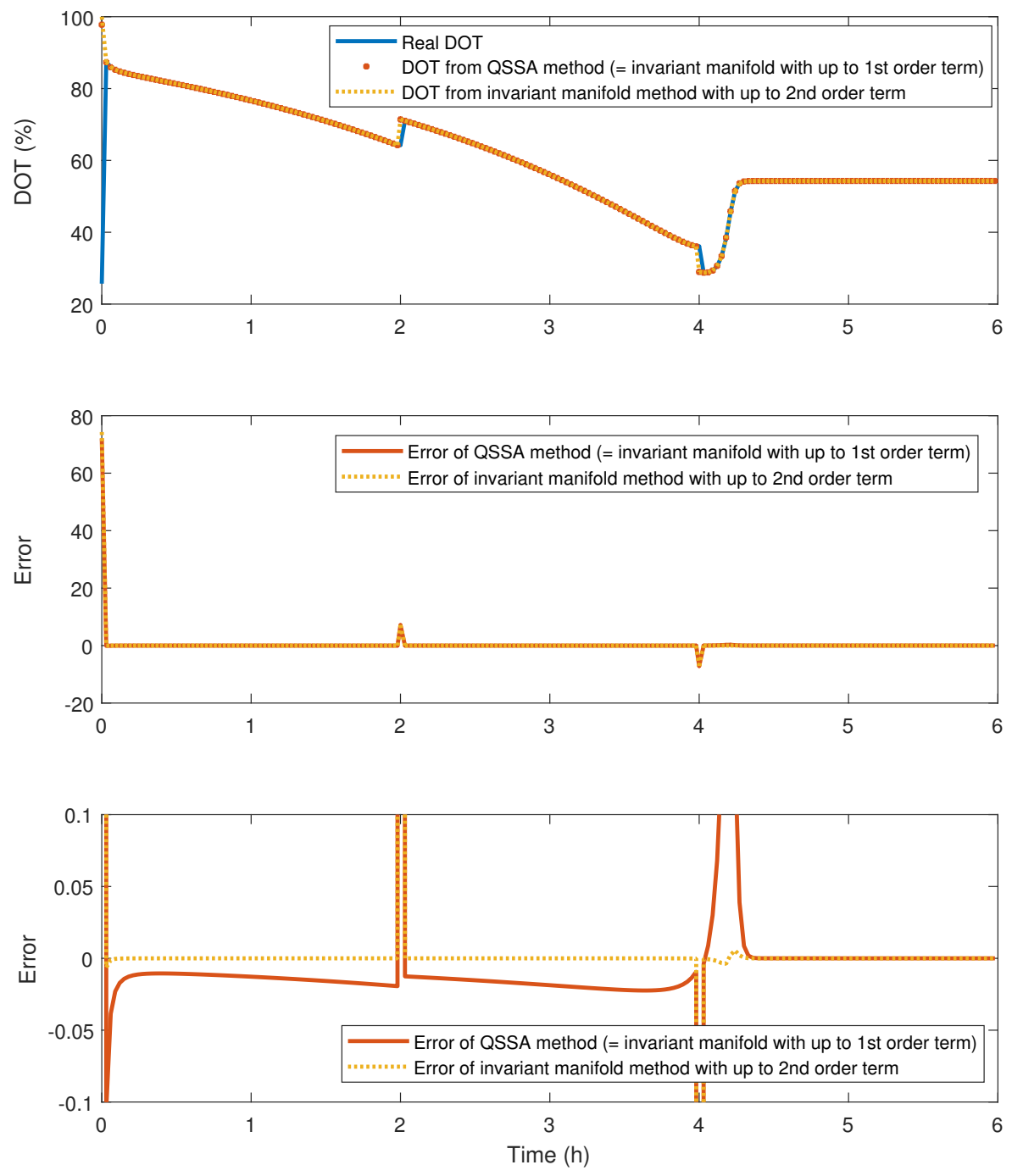

Figure 2: Simulation of the basic continuous aerobic process

in Figure 4. While the invariant manifold with up to the second order term still show good accuracy, one can notice the significant error between the QSSA approximation with the exact one, especially in the transient phase.

The reduced model for batch processes can be treated as a simplified version of the continuous process with $D=0$. 

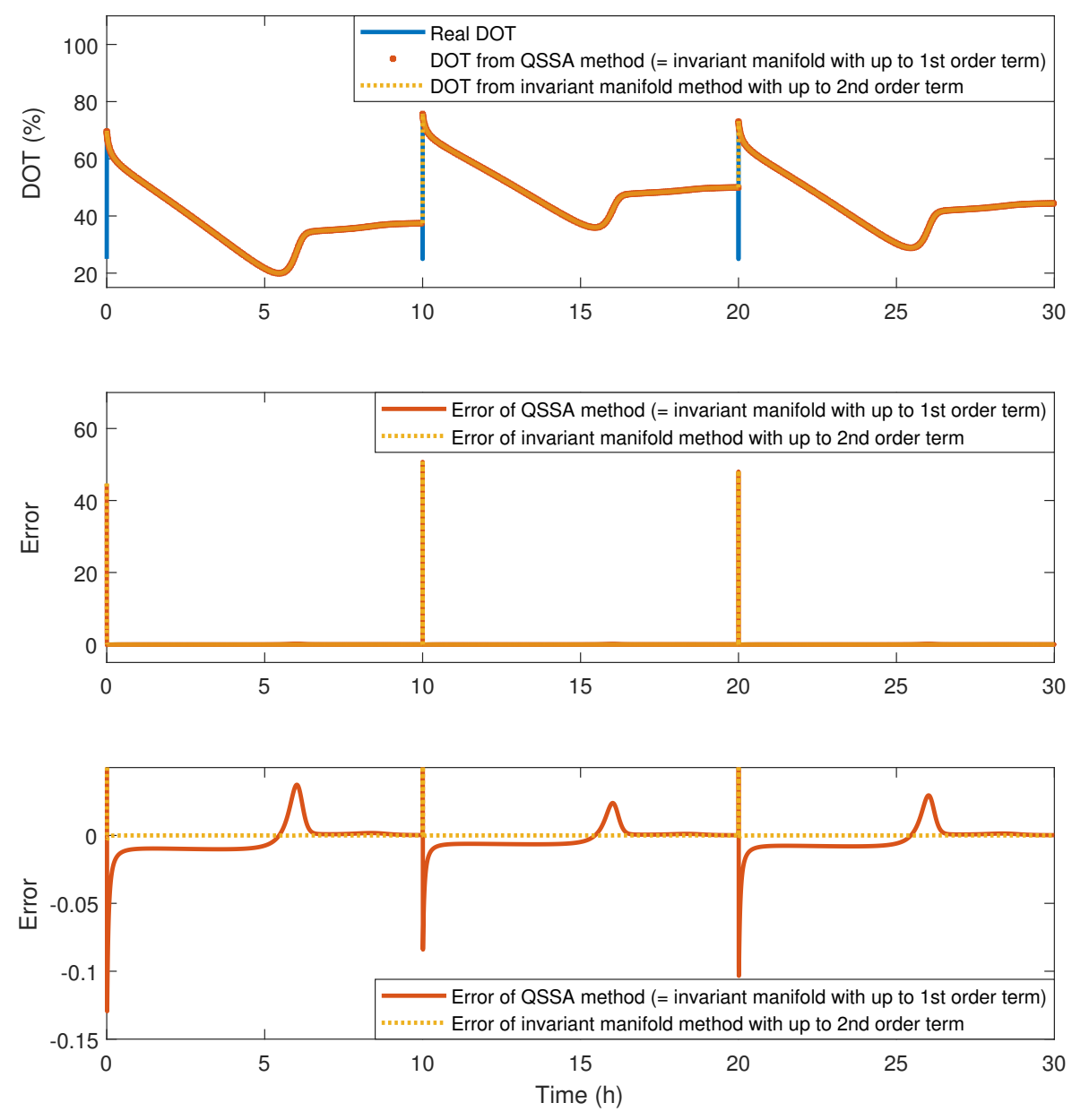

Figure 3: Simulation of the E. Coli continuous process

\subsection{Fed-Batch}

It has been demonstrated that the slow-motion invariant manifold could be used to approximate the fast state locally around the steady state. For the fed-batch operation, a local analysis is no longer meaningful. Therefore, in order to apply a similar method, we need to also show that the calculated slowmotion invariant manifold would asymptotically approach the real state under fed-batch operations over the entire feasible domain. By setting $F_{\text {out }}=0$ in (1), 

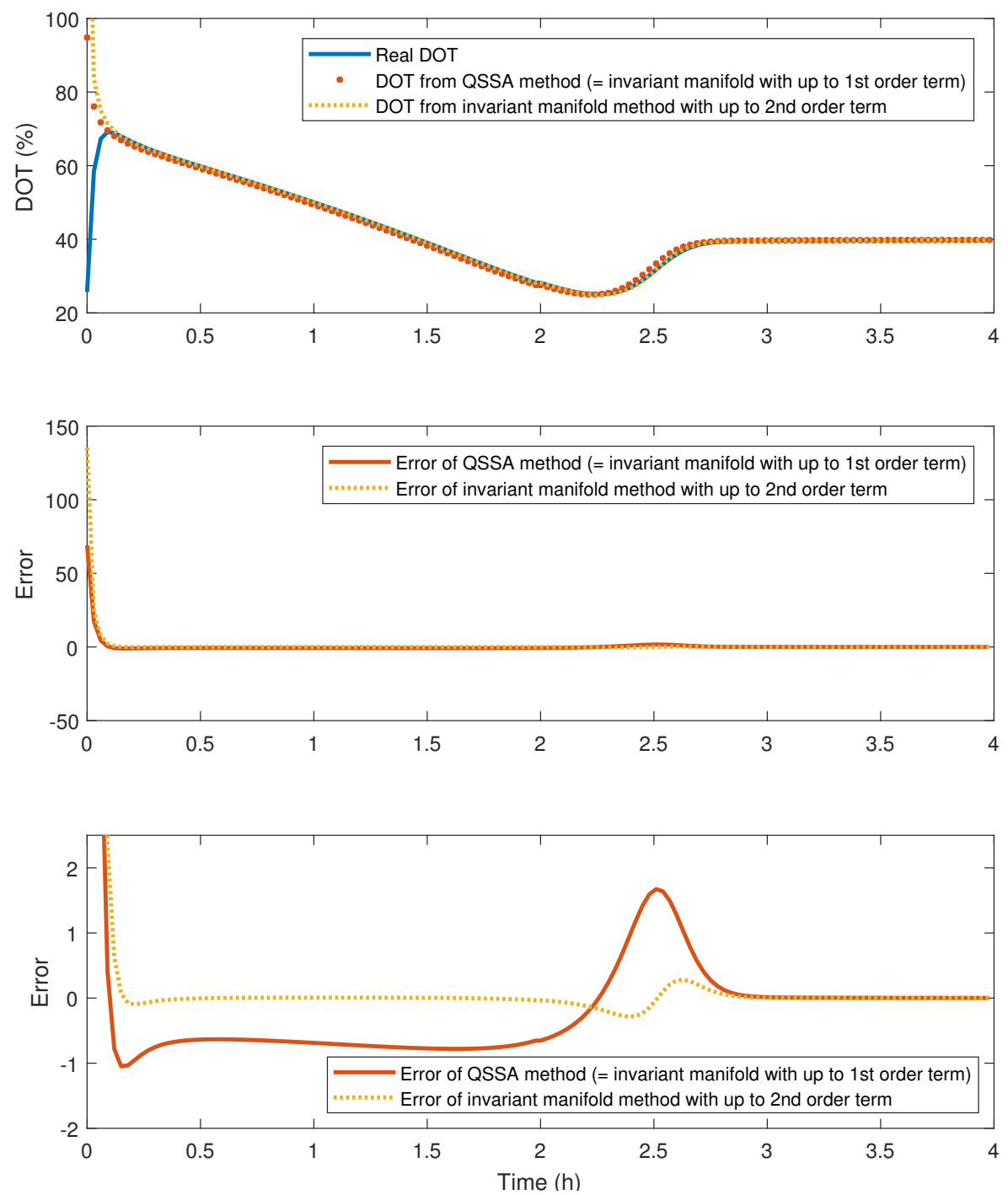

Figure 4: Simulation of an arbitrary continuous process with small $K_{L} a$

the fed-batch process can be described as follows:

$$
\begin{aligned}
\frac{d S}{d t} & =-\frac{F}{V}\left(S-S_{i n}\right)-q_{s} X \\
\frac{d X}{d t} & =-\frac{F}{V} X+\mu X \\
\frac{d D O T}{d t} & =K_{L} a\left(D O T^{*}-D O T\right)-H q_{o} X \\
\frac{d V}{d t} & =F
\end{aligned}
$$


To calculate the slow invariant manifold, we follow the same procedure as for the continuous process. The corresponding slow-motion invariant manifold equation for system $(14)$ is:

$$
\begin{array}{r}
\frac{\partial T(S, X)}{\partial X}\left(-\frac{F}{V} X+\mu(S) X\right)+\frac{\partial T(S, X)}{\partial S}\left\{\frac{F}{V}\left(S_{i n}-S\right)-q_{s}(S) X\right\}+\frac{\partial T(S, X)}{\partial V} F \\
=K_{L} a\left(D O T^{*}-T(S, X)\right)-q_{o}(S) H X
\end{array}
$$

Similarly, define $\epsilon=1 / K_{L} a$ as the small parameter. Dividing both sides of the equation by $K_{L} a$, we have

$$
\begin{aligned}
\varepsilon \frac{\partial T(S, X)}{\partial X}\left(-\frac{F}{V} X+\right. & \mu(S) X)+\varepsilon \frac{\partial T(S, X)}{\partial S}\left[\frac{F}{V}\left(S_{i n}-S\right)-q_{s}(S) X\right] \\
& +\varepsilon \frac{\partial T(S, X)}{\partial V} F=D O T^{*}-T(S, X)-\varepsilon q_{o}(S) H X
\end{aligned}
$$

Expanding $T(S, X)$ in asymptotic series in terms of the small parameter, substituting the expansion for $T(S, X)$ into the equation $(16)$, and solving the equation, we will get the slow invariant manifold as:

$$
\begin{aligned}
\operatorname{DOT}(X, S, V) & =P_{0}(X, S, V)+\frac{P_{1}(X, S, V)}{K_{L} a}+\frac{P_{2}(X, S, V)}{K_{L} a^{2}} \ldots \\
& =D O T^{*}-\frac{X H Y_{o} q_{s} \max }{K_{L} a} \frac{S}{S+K_{s}} \\
& +\frac{1}{K_{L} a^{2}}\left\{q_{o} H\left(-\frac{F}{V} X+\mu(S) X\right)+Y_{o} X H \frac{d q_{s}}{d S}\left[\frac{F}{V}\left(-S-S_{i n}\right)+q_{s}(S) X\right]\right\} \\
& +O\left[\left(\frac{1}{K_{L} a}\right)^{2}\right]
\end{aligned}
$$

Note that this result is very similar to that of the continuous model, except for the substitution of $D$ with $F / V$. However, one may notice a significant difference between them when higher order terms are calculated.

Next we will show that this calculated slow invariant manifold will asymptotically approach the real state.

Proposition. Let $D O T^{\prime}(S(t), X(t), V(t))$ be the unique solution of the invariance equation 15 for the model (1), and $(S(t), X(t), D O T(t), V(t))$ a solution curve of model (11). The dynamics of the off-manifold coordinate $z=$ $D O T(t)-D O T^{\prime}(S(t), X(t), V(t))$ decays exponentially and the rate of decay is governed by $K_{L} a$. 
Proof. Since $D O T^{\prime}$ is the solution of Equation (15), it holds that:

$$
\begin{aligned}
& \frac{\partial D O T^{\prime}(S, X, V)}{\partial S} f_{1}(S, X, V)+\frac{\partial D O T^{\prime}(S, X, V)}{\partial X} f_{2}(S, X, V)+\frac{\partial D O T^{\prime}(S, X, V)}{\partial V} F \\
& =-K_{L} a \cdot D O T^{\prime}+K_{L} a \cdot D O T^{*}-Y_{o} q_{s \max } X H \frac{S}{S+K_{S}}
\end{aligned}
$$

The dynamics of $z=D O T-D O T^{\prime}$ is described by the following nonlinear differential equation

$$
\begin{aligned}
\frac{d z}{d t} & =\frac{d D O T}{d t}-\frac{d D O T^{\prime}}{d t} \\
& =\left(-K_{L} a \cdot D O T-Y_{o} q_{s \max } \frac{S}{S+K_{S}} X H+K_{L} a \cdot D O T^{*}\right) \\
& -\left(\frac{\partial D O T^{\prime}(S, X, V)}{\partial S} f_{1}(S, X, V)+\frac{\partial D O T^{\prime}(S, X, V)}{\partial X} f_{2}(S, X, V)+\frac{\partial D O T^{\prime}(S, X, V)}{\partial V} F\right) \\
& =\left(-K_{L} a \cdot D O T-Y_{o} q_{s \max } X H \frac{S}{S+K_{S}}\right)-\left(-K_{L} a \cdot D O T^{\prime}-Y_{o} q_{s \max } X H \frac{S}{S+K_{S}}\right) \\
& =-K_{L} a \cdot z
\end{aligned}
$$

So the off-manifold coordinate will exponentially decay to 0 with the rate of $K_{L} a$.

The result calculated from quasi-steady-state approximation remains the same as the one for continuous model. That's because the first two terms contain no dilution-related terms.

For the E. coli model (5), the fed-batch result also turns out to be similar with the continuous one. The reader can refer to the reduced continuous $E$. coli model 13 and substitute the dilution rate $D$ with $F / V$ for up to the second order terms to find the reduced fed-batch model.

In Figure 5 , the same simulation as in Figure 2 is done for the basic aerobic process model under fed-batch operation. It can be noted that both the reduced models derived using slow-motion invariant manifold and from QSSA method have similar performance as the continuous model. We skip the simulation result for fed-batch $E$. coli model as it shows similar behaviour as the continuous case. 

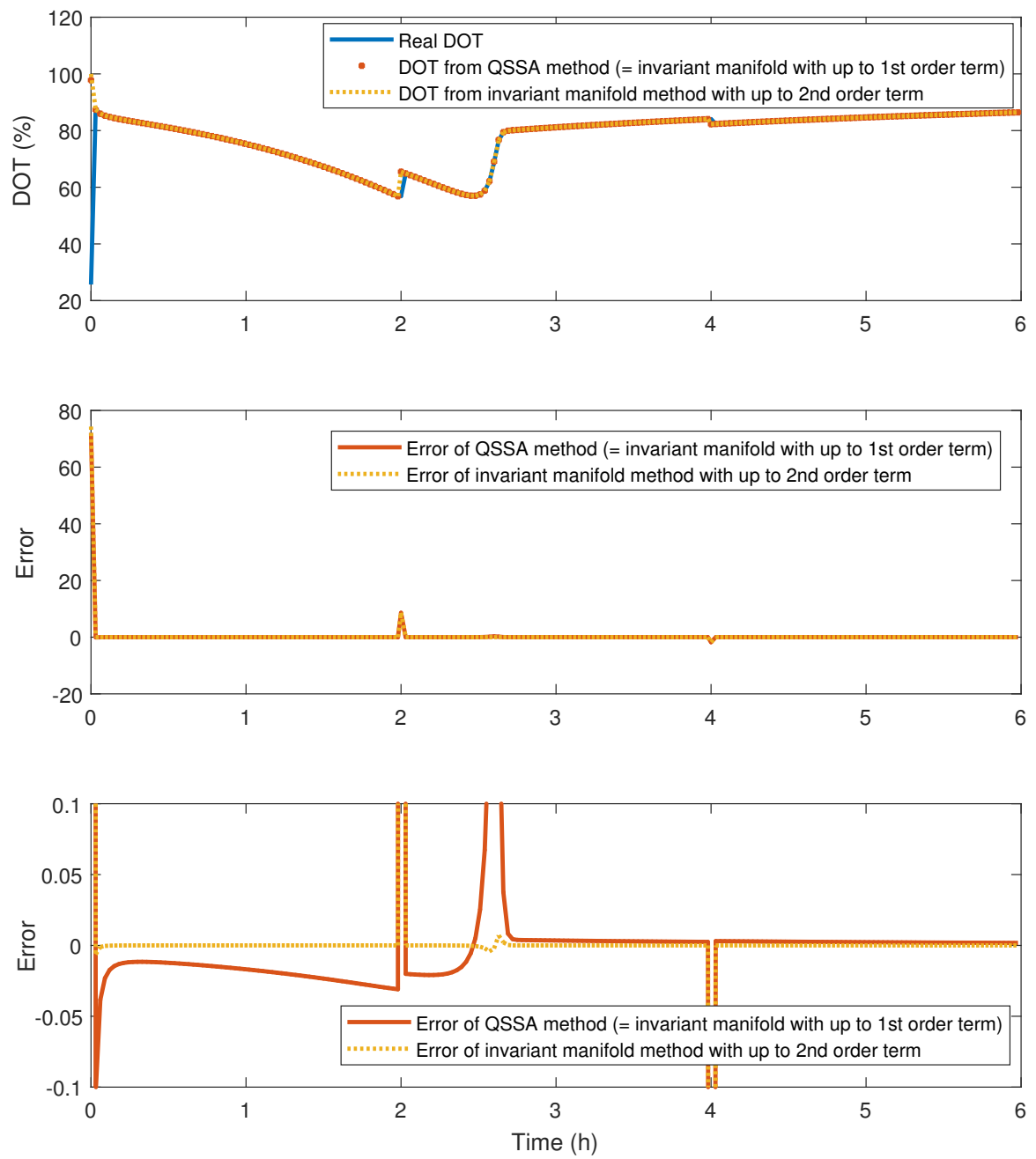

Figure 5: Simulation of the fed-batch process under substrate limited condition

\section{Application of Reduced Model: Simplified Observer Design for Aerobic \\ Process Monitoring}

The lack for affordable and reliable sensors that can measure all states on-line poses challenges to the bioreactor operation. It also makes advanced monitoring and control for these processes a tough problem. For this reason, an observer, also known as a soft sensor, becomes useful as it could estimate some inaccessible state information and unknown parameter values based on the measured 
variables with certain algorithms. Common observer techniques include the Luenberger observer 34] and the Kalman filter [35]. There are a number of published books and articles that give introduction on the theory and their application on bioreactors [36, 37, 38, 39]. In this part, we will use the Luenberger for the fed-batch system.

For the basic 4-state fed-batch aerobic process, the dissolved oxygen tension $D O T$ and the volume of culture are usually measured continuously on-line, but the biomass and the substrate concentration $X$ and $S$ are either hard to 230 measure or can only be measured offline with a relatively slow sampling rate due to the sensor limitation. And the value of inlet flow rate $F$ might be hard to be accurately measured in some applications. In this case, we assume the measurements to be available for DOT and $X$, and the estimation for state variable $S$ is needed. Also, the inlet flow rate $F$ is piecewise time variant and 235 unknown.

At first, we consider all the measurements to be continuous. The traditional observer is a full-order constant-gain Luenberger observer with a parameter 
estimator as follow:

$$
\frac{d}{d t}\left[\begin{array}{c}
\hat{S} \\
\hat{X} \\
D \hat{O} T \\
\hat{V} \\
\hat{F}
\end{array}\right]=\left[\begin{array}{c}
-\hat{F}\left(\hat{S}-S_{i n}\right)-\hat{X} q_{s}(\hat{S}, D \hat{O} T) \\
-\frac{\hat{F}}{\hat{X}}+Y_{x} \hat{X} q_{s}(\hat{S}, D \hat{O} T) \\
K_{L} a\left(D O T^{*}-D \hat{O} T\right)-H Y_{o} \hat{X} q_{s}(\hat{S}, D \hat{O} T) \\
\hat{F} \\
0
\end{array}\right]+L\left[\begin{array}{c}
X-\hat{X} \\
D O T-D \hat{O} T \\
V-\hat{V}
\end{array}\right]
$$

where $L$ is designed using classical pole placement methods.

For the reduced model, with the measurement of $X, D O T$ and $V$, unknown inlet flow rate $F$ can be obtained by calculating the time derivative of $V$, and $S$ can be solved directly from the algebraic equation (17) by substituting $X$, $D O T$ and $V$ with the measured data at every sampling point. To simulate this process, we use the parameter values in Table 1 . The initial culture volume $V=0.2 \mathrm{~L}$, and the initial $F=0.1 \mathrm{~L} / \mathrm{h} . F$ is stepped up to $0.12,0.16,0.2 \mathrm{~L} / \mathrm{h}$ at times 1,2 and 3 . We plot the substrate concentration $S$ from the simulated process (blue line), from the Luenberger observer (red dash line) and from the algebraic equation (green line) in the same graph in Figure 6. We can tell that both the observer and the solution from the function are in great agreement with the exact $S$ and $F$ during the entire time period, as they are nearly on top of each other.

Next, we consider the measurement of $X$ to be discrete, sampled at every 0.2 hour. There are several methods established to deal with discrete-time state estimation problems 40, 41, 42, 43. To improve accuracy in the presence of large sampling periods, one could use a predictor $w$ to estimate $X$ between sampling points where there is no measurement 44]. At each sampling time point, $w$ is reset to be the measurement of $X$. And between sampling points, it 255 is simulated under the same dynamics of $X$. This method is demonstrated by the diagram of Figure 7 

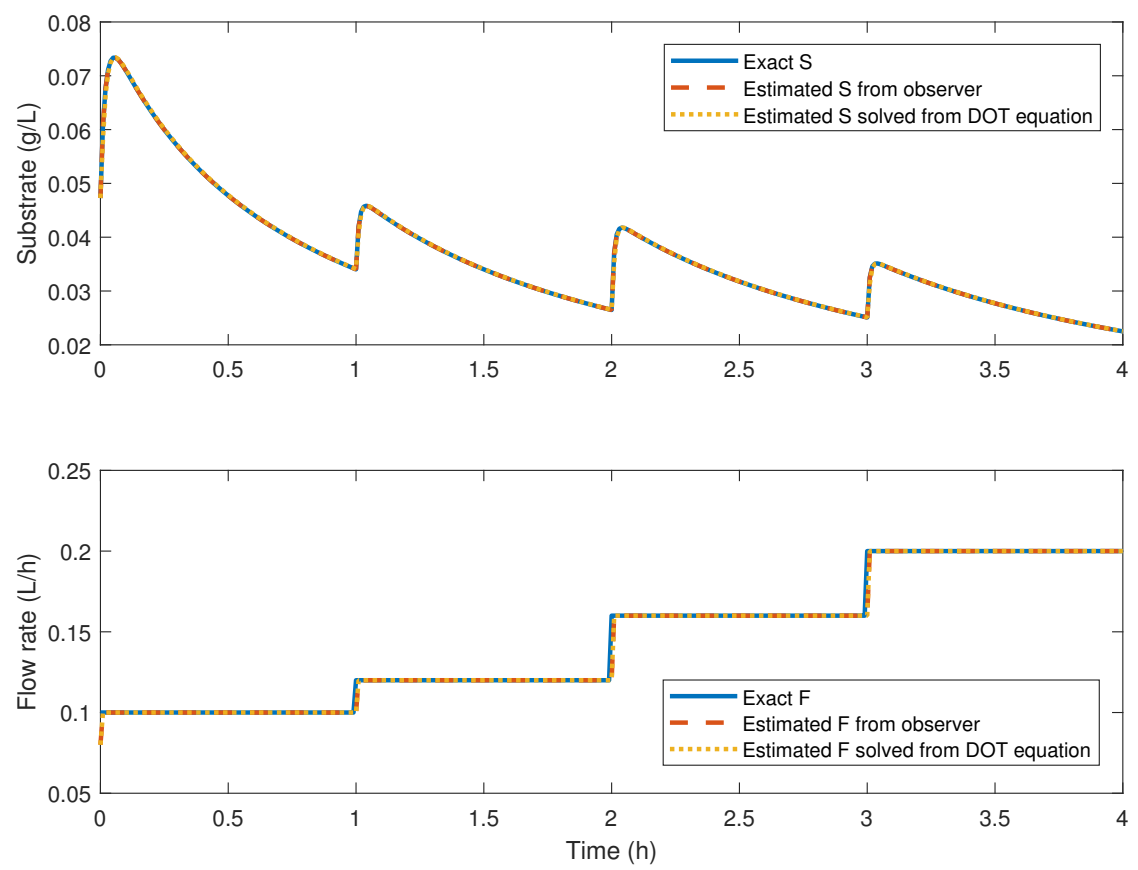

Figure 6: State estimation with continuous measurement

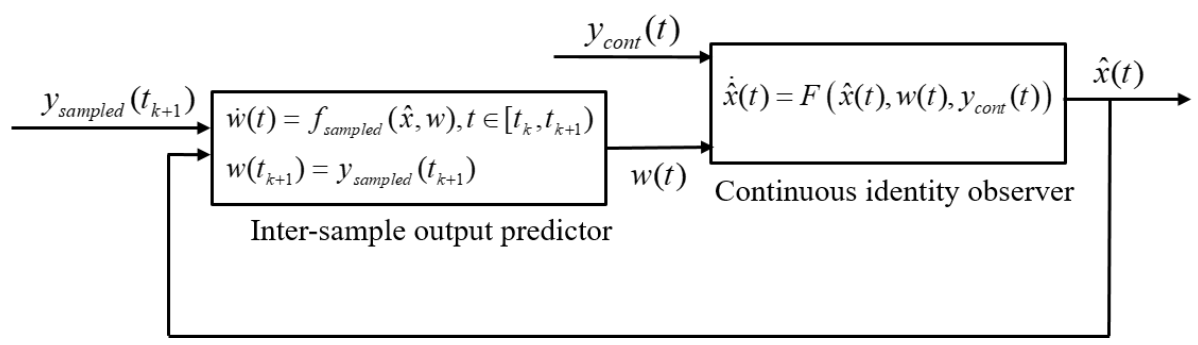

Figure 7: Observer design scheme with inter sample predictor

With the predictor, the full order observer becomes

$\frac{d}{d t}\left[\begin{array}{c}\hat{S} \\ \hat{X} \\ D \hat{O} T \\ \hat{V} \\ \hat{F}\end{array}\right]=\left[\begin{array}{c}-\frac{\hat{F}}{\hat{V}}\left(\hat{S}-S_{i n}\right)-\hat{X} q_{s}(\hat{S}, D \hat{O} T) \\ -\frac{\hat{F}}{\hat{X}}+Y_{x} \hat{X} q_{s}(\hat{S}, D \hat{O} T) \\ K_{L} a\left(D O T^{*}-D \hat{O} T\right)-H Y_{o} \hat{X} q_{s}(\hat{S}, D \hat{O} T) \\ \hat{F} \\ 0\end{array}\right]+L\left[\begin{array}{c}w-\hat{X} \\ D O T-D \hat{O} T \\ V-\hat{V}\end{array}\right]$ 


$$
\begin{aligned}
\frac{d w}{d t} & =-\frac{\hat{F}}{\hat{V}} w+Y_{x} w q_{s}(\hat{S}, D \hat{O} T), t \in\left[t_{k}, t_{k+1}\right) \\
w\left(t_{k+1}\right) & =X\left(t_{k+1}\right), t=t_{k+1}
\end{aligned}
$$

For the reduced model, $\mathrm{S}$ would be solved from the algebraic equation 17 as $D O T=T(w, S, V)$, where $w$ is the solution of

$$
\left\{\begin{array}{l}
\frac{d w}{d t}=-\frac{F}{V} w+Y_{x} w q_{s}\left(S_{\text {solved }}, D O T\right), t \in\left[t_{k}, t_{k+1}\right) \\
w\left(t_{k+1}\right)=X\left(t_{k+1}\right), t=t_{k+1}
\end{array}\right.
$$

From Figure 8, we observe that both methods show good performance in estimating the state $S$ and parameter value $F$.
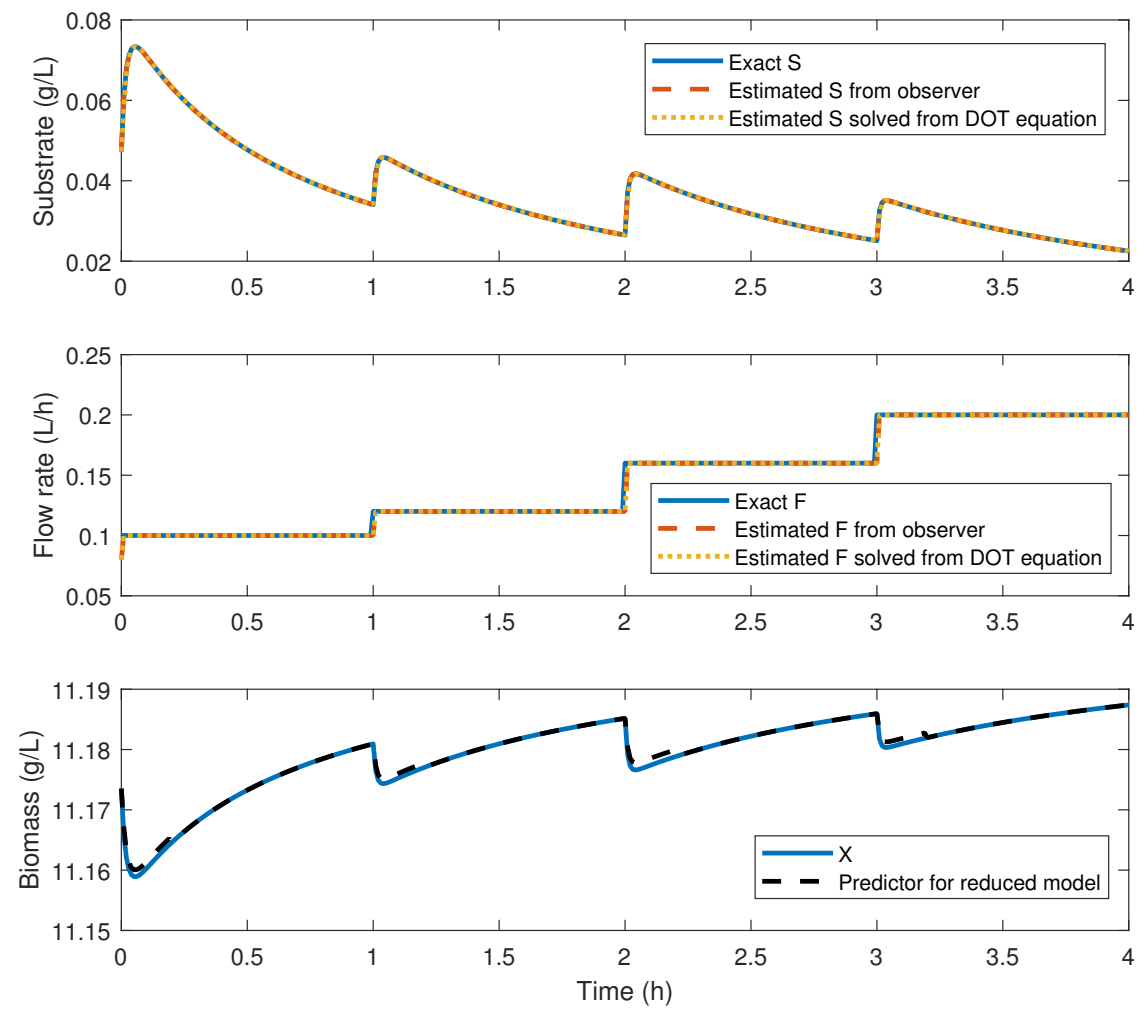

Figure 8: State estimation with continuous DOT measurement and sampled X measurement 


\section{Discussion}

260 cesses. For the very basic aerobic model, the analysis shows that the process typically consists of both fast and slow dynamics. Considering that the process is substrate-limited in practice, the slow and fast dynamics are connected in series and the dissolved oxygen tension is the fast state associated with the fast the aforementioned property. This is the theoretical basis that motivates the model reduction to eliminate the fast dynamics associated with the state DOT.

To reduce the model, we use the slow-motion invariant manifold to approximate the fast state DOT, by assuming the fast dynamics to be instantaneous. the real state once the fast dynamics dies out. To find the slow invariant manifold, a partial differential equation is approximately solved via perturbation analysis and the accuracy of the solution can be adjusted via the truncation order. As a comparison, the quasi steady state approximation, which is not done to both continuous and fed-batch processes.

Finally, an observer design problem is studied as an example to show how this reduced model could be useful in bioengineering applications. With the reduced model, the traditional observer design problem can be simplified, leading to a

rate measurement problem can also be handled with the introduction of an inter-sample predictor. And this zero-order observer shows great performance in estimating unmeasured states and unknown parameters. 


\section{Conclusion} in the rate of change of their modes. This can be used as the basis to increase the tractability of differential equation models that describe processes governed by both fast and slow dynamics simultaneously. As we have demonstrated in this work, the stiffness of aerobic macro-kinetic models can be significantly reduced

\section{Acknowledgments}

Zhaoyang Duan and Costas Kravaris acknowledge financial support from the National Science Foundation through grant CBET-1706201.

\section{References}

315

[1] A. Berlec, B. Štrukelj, Current state and recent advances in biopharmaceutical production in escherichia coli, yeasts and mammalian cells, Journal of industrial microbiology \& biotechnology 40 (3-4) (2013) 257-274. 
[2] P. Neubauer, M. N. Cruz Bournazou, F. Glauche, S. Junne, A. Knepper, M. Raven, Consistent development of bioprocesses from microliter cultures to the industrial scale, Engineering in Life Sciences 13 (3) (2013) 224-238.

[3] M. Gavrilescu, Y. Chisti, Biotechnologya sustainable alternative for chemical industry, Biotechnology Advances 23 (7-8) (2005) 471-499.

[4] H. Rabitz, M. Kramer, D. Dacol, Sensitivity analysis in chemical kinetics, Annual review of physical chemistry 34 (1) (1983) 419-461.

[5] R. Wang, P. Unrean, C. J. Franzén, Model-based optimization and scaleup of multi-feed simultaneous saccharification and co-fermentation of steam pre-treated lignocellulose enables high gravity ethanol production, Biotechnology for Biofuels 9 (1) (2016) 88.

[6] J. O. Westman, C. J. Franzén, Current progress in high cell density yeast bioprocesses for bioethanol production, Biotechnology journal 10 (8) (2015) $1185-1195$.

[7] R. Wang, R. Koppram, L. Olsson, C. J. Franzén, Kinetic modeling of multifeed simultaneous saccharification and co-fermentation of pretreated birch to ethanol, Bioresource Technology 172 (2014) 303-311.

[8] E. Anane, D. C. López C, P. Neubauer, M. N. Cruz Bournazou, Modelling overflow metabolism in Escherichia coli by acetate cycling, Biochemical Engineering Journal 125 (2017) 23-30.

[9] E. Anane, Á. C. García, B. Haby, S. Hans, N. Krausch, M. Krewinkel, P. Hauptmann, P. Neubauer, M. N. Cruz Bournazou, Model-based framework for parallel scale down fed-batch cultivations in mini-bioreactors for accelerated phenotyping, Biotechnology and bioengineering.

[10] J. Möller, K. B. Kuchemüller, T. Steinmetz, K. S. Koopmann, R. Pörtner, Model-assisted design of experiments as a concept for knowledge-based bioprocess development, Bioprocess and biosystems engineering (2019) 1-16. 
[12] C. A. Floudas, C. E. Gounaris, A review of recent advances in global optimization, Journal of Global Optimization 45 (1) (2009) 3-38.

[13] M. Koutinas, A. Kiparissides, E. N. Pistikopoulos, A. Mantalaris, Bioprocess systems engineering: transferring traditional process engineering prin-

[19] L. A. Segel, M. Slemrod, The quasi-steady-state assumption: a case study in perturbation, SIAM review 31 (3) (1989) 446-477.

[20] A. Gorban, Model reduction in chemical dynamics: slow invariant manifolds, singular perturbations, thermodynamic estimates, and analysis of ciples to industrial biotechnology, Computational and structural biotechnology journal 3 (4) (2012) e201210022.

[14] A. Psaltis, I. K. Kookos, C. Kravaris, Plant-wide control structure selection methodology based on economics, Computers \& Chemical Engineering 52 (2013) 240-248.

[15] L. Mears, S. M. Stocks, M. O. Albaek, G. Sin, K. V. Gernaey, Mechanistic Fermentation Models for Process Design, Monitoring, and Control, Trends in Biotechnology 35 (10) (2017) 914-924.

[16] S.-O. Enfors, L. Häggström, Bioprocess technology: fundamentals and applications, Royal Institute of Technology, 2000.

[17] M. N. Cruz Bournazou, H. Arellano-Garcia, G. Wozny, G. Lyberatos, C. Kravaris, Asm3 extended for two-step nitrification-denitrification: a model reduction for sequencing batch reactors, Journal of Chemical Technology \& Biotechnology 87 (7) (2012) 887-896.

[18] M. S. Okino, M. L. Mavrovouniotis, Simplification of mathematical models of chemical reaction systems, Chemical reviews 98 (2) (1998) 391-408. reaction graph, Current Opinion in Chemical Engineering 21 (2018) 48-59. 
[21] P. V. Kokotovic, R. E. O’Malley Jr, P. Sannuti, Singular perturbations and order reduction in control theoryan overview, Automatica 12 (2) (1976) $123-132$.

[22] A. Kumar, P. D. Christofides, P. Daoutidis, Singular perturbation modeling of nonlinear processes with nonexplicit time-scale multiplicity, Chemical Engineering Science 53 (8) (1998) 1491-1504.

[23] N. Kazantzis, C. Kravaris, L. Syrou, A new model reduction method for nonlinear dynamical systems, Nonlinear Dynamics 59 (1-2) (2010) 183.

[24] Z. Duan, M. N. Cruz Bournazou, C. Kravaris, Dynamic model reduction for two-stage anaerobic digestion processes, Chemical Engineering Journal 327 (2017) 1102-1116.

[25] Z. Duan, C. Kravaris, Robust stabilization of a two-stage anaerobic bioreactor system, in: 2017 IEEE 56th Annual Conference on Decision and Control (CDC), IEEE, 2017, pp. 2083-2088.

[26] Z. Duan, C. Kravaris, Robust stabilization of a two-stage continuous anaerobic bioreactor system, AIChE Journal 64 (4) (2018) 1295-1304.

[27] K. Stamatelatou, L. Syrou, C. Kravaris, G. Lyberatos, An invariant manifold approach for cstr model reduction in the presence of multi-step biochemical reaction schemes. application to anaerobic digestion, Chemical Engineering Journal 150 (2-3) (2009) 462-475.

[28] M. R. Roussel, S. J. Fraser, Invariant manifold methods for metabolic model reduction, Chaos: An Interdisciplinary Journal of Nonlinear Science 11 (1) (2001) 196-206.

[29] A. N. Gorban, I. V. Karlin, Method of invariant manifold for chemical kinetics, Chemical Engineering Science 58 (21) (2003) 4751-4768.

[30] A. N. Gorban, I. V. Karlin, Invariant manifolds for physical and chemical kinetics, Vol. 660, Springer Science \& Business Media, 2005. 
[31] E. Chiavazzo, A. N. Gorban, I. V. Karlin, Comparison of invariant manifolds for model reduction in chemical kinetics, Commun. Comput. Phys 2 (5) (2007) 964-992.

[32] B. Haby, F. Glauche, S. Hans, M. N. Cruz Bournazou, P. Neubauer, Stammcharakterisierung mittels on-line-redesign von experimenten, BIOspektrum 24 (1) (2018) 39-42.

[33] P. Neubauer, M. N. Cruz Bournazou, F. Glauche, S. Junne, A. Knepper, M. Raven, Consistent development of bioprocesses from microliter cultures to the industrial scale, Engineering in Life Sciences 13 (3) (2013) 224-238.

[34] D. G. Luenberger, Observing the state of a linear system, IEEE transactions on military electronics 8 (2) (1964) 74-80.

[35] G. Welch, G. Bishop, et al., An introduction to the kalman filter.

[36] N. Kazantzis, C. Kravaris, Nonlinear observer design using lyapunovs auxiliary theorem, Systems \& Control Letters 34 (5) (1998) 241-247.

[37] C. Kravaris, V. Sotiropoulos, C. Georgiou, N. Kazantzis, M. Xiao, A. J. Krener, Nonlinear observer design for state and disturbance estimation, in: Proceedings of the 2004 American Control Conference, Vol. 4, IEEE, 2004, pp. 2931-2936.

[38] C. Kravaris, J. Hahn, Y. Chu, Advances and selected recent developments in state and parameter estimation, Computers \& chemical engineering 51 (2013) 111-123.

[39] D. Dochain, State and parameter estimation in chemical and biochemical processes: a tutorial, Journal of process control 13 (8) (2003) 801-818.

[40] S. Tatiraju, M. Soroush, B. A. Ogunnaike, Multirate nonlinear state estimation with application to a polymerization reactor, AIChE Journal 45 (4) (1999) 769-780. 
[41] C. Ling, C. Kravaris, Multi-rate observer design for process monitoring using asynchronous inter-sample output predictions, AIChE Journal 63 (8) (2017) 3384-3394.

[42] C. Ling, C. Kravaris, Multi-rate sampled-data observers based on a continuous-time design, in: 2017 IEEE 56th Annual Conference on Decision and Control (CDC), IEEE, 2017, pp. 3664-3669.

[43] A. Liu, W.-a. Zhang, L. Yu, J. Chen, Moving horizon estimation for multirate systems, in: 2015 54th IEEE Conference on Decision and Control (CDC), IEEE, 2015, pp. 6850-6855.

[44] C. Ling, C. Kravaris, Multi-rate sampled-data observers based on a continuous-time design, in: 2017 IEEE 56th Annual Conference on Decision and Control (CDC), IEEE, 2017, pp. 3664-3669. 